
\title{
Research Suate \\ The Effect of Noise-Induced Quantum Coherence in the Intermediate Band Solar Cells
}

Mohsen Daryani ( $\nabla$ mohsen.daryani@gmail.com )

Islamic Azad University

Ali Rostami

University of Tabriz https://orcid.org/0000-0002-8727-4711

Gaffar Darvish

Islamic Azad University

Mohammad Kazem Morravej Farshi

Tarbiat Modares University

\section{Research Article}

Keywords: Fano interference, Intermediate-band solar cells, Nano-structure solar cells, Quantum coherence.

Posted Date: June 17th, 2021

DOI: https://doi.org/10.21203/rs.3.rs-552022/v1

License: (c) (1) This work is licensed under a Creative Commons Attribution 4.0 International License.

Read Full License 


\title{
The Effect of Noise-Induced Quantum Coherence in the Intermediate Band Solar Cells
}

\author{
M. Daryani ${ }^{1}$, A. Rostami ${ }^{2,3}$, G. Darvish ${ }^{1}$, M. K. Morravej Farshi ${ }^{4}$ \\ ${ }^{1}$ Department of Electrical Engineering, Sciences and Research Branch, Islamic Azad University, Tehran 1477893855, Iran. \\ ${ }^{2}$ Photonics and Nanocrystals Research Lab., (PNRL), Faculty of Electrical and Computer Engineering, University of Tabriz, Tabriz \\ 5166614761, Iran
}

${ }^{3}$ SP-EPT Lab., ASEPE Company, Industrial Park of Advanced Technologies, Tabriz, Iran

${ }^{4}$ Faculty of Electrical \& Computer Engineering, Tarbiat Modares University, Tehran 1411713116, Iran

\begin{abstract}
It has been shown that quantum coherence induced by incoherent light can increase the efficiency of solar cells. Here we evaluate the effect of such coherence in the intermediate band solar cells. We first examine a six-level quantum IBSC model and demonstrate by simulation that the maximum of output power in a solar cell with quantum structure increases more than 16 percent in the case of coherence existence. We then propose an IBSC model which can absorb continuous spectra of sunlight and show that the quantum coherence can increase the output power of the cell. For instance, calculations indicate that the coherence makes an increase of about $31 \%$ in the maximum output power of a cell that the width of the conduction and intermediate bands are 100 and $10 \mathrm{meV}$, respectively. Also, our calculations show that the quantum coherence effect is still observed in increasing the solar cell power by expanding the width of the conduction band, although the output power is reduced due to increase in the thermalization loss. However, expanding the width of the intermediate band reduces the coherence effect.
\end{abstract}

Index Terms — Fano interference, Intermediate-band solar cells, Nano-structure solar cells, Quantum coherence.

\section{INTRODUCTION}

The efficiency of single band gap solar cells has continuously increased in recent years and is now approaching the ShockleyQueisser conversion efficiency limit [1]. This fundamental limit for single junction devices operating under 1-sun illumination is about $31 \%$. It is substantially due to the fact that only the photons of energy greater than the energy band gap are absorbed. These photons create electron-hole pairs which their excess energy is wasted by emitting phonons. The photons that have less energy than the energy band gap are not absorbed [2]. Attempts to overcome this limitation have led to the development of many high-efficiency solar cell concepts, including multi-junction, intermediate band, and hot-carrier solar cells, multiple exciton generations, and up- and down-conversion [3]. Multi-junction solar cells have already broken this limitation [1], but because of their high price and demanding material growth, it is desirable to find other ways to high efficiency. The other solution that has been proposed is that of intermediate band solar cells (IBSCs). In an IBSC, a set of allowed electronic states, called intermediate band (IB), is introduced within the semiconductor band gap [4]. This provides new routes to the carriers that besides photons with energy above the band gap, two lower energy photons can promote electrons from the valence to the conduction band through the intermediate levels, thus these two sub-gap photons can create an additional electron-hole pair, which can be collected at a voltage that is limited by the host large band gap. Consequently, the addition of the IB increases the light-to-current conversion efficiency of the solar cell. An additional condition to ensure such an efficiency enhancement is that IB needs to be optically connected to the valence band but electrically isolated from the other bands [5]. It has been shown [4] that the maximum theoretical conversion efficiency of a solar cell with a single IB reaches about $46.8 \%, 63.2 \%$ under 1-Sun and fullconcentration, respectively, each about 50\% (relative) higher than for a single-junction solar cell [6]. Also, studies have shown that increasing the number of IBs provides a greater increase in efficiency [7, 8]. Although experimental studies have demonstrated the key IBSC operating principles [9], [10], it is challenging to find suitable IB materials in bulk semiconductors [11]. Several different methods have been proposed for creating intermediate energy states, for instance, highly mismatched semiconductor alloys [12], impurities in the semiconductor band gap [13], and quantum structures [14]. Among them, the implementation of quantum dots (QDs) in a host semiconductor is the one that has verified most of the phenomena expected in IBSC operation [15]. In recent years, various aspects of quantum dot intermediate band solar cells have been studied. In particular, cell fabrication, materials, performance and charge transport [16]-[21] are the subjects of recent investigation. Although the experimental results for efficiency in intermediate band solar cells are very low compared to the theoretically 
calculated value, there seems to be a promising prospect [15]. One of the main challenges in QD-based IB solar cells is to overcome low absorption between the valence and the IB transitions [11].

On the other hand, it has been shown that quantum coherence has a significant role in modifying photon absorption and emission profiles. The effect of quantum coherence has been studied in many events such as lasing without inversion [22],

electromagnetically induced transparency [23] and slow light [24], [25] in atomic systems. Also, it has been demonstrated that coherence can also play a role in semiconductor quantum dots [26], [27] and heterostructures [28]. It has also been shown that quantum coherence can be used to break the detailed balance and increase the photocurrent of a photocell [29]-[32]. There are two main mechanisms to generate coherence, the first one is generating coherence by external incoherent field [33], and the other mechanism is the generation of the coherence by incoherent processes such as spontaneous emission, as shown by Agarwal in [34]. In particular, the possibility of generating quantum coherence via the interaction of multilevel quantum systems with incoherent light has recently attracted much interest [30], [32], [35]-[36]. This noise-induced coherence are created via quantum interference of the transition amplitudes leading to the same final state and called Fano interference [37]. It has been shown that coherence produced by both methods can enhance absorption of light photons in a photocell, and lead to increase of the cell power [29], [31], [38]-[40]. Also, in a model of the IBSC with two energy levels in the band gap, It has been shown that the output power of the cell can be increased by noise-induced coherence between the intermediate levels [41]. We have already shown that the coherence in solar cells with continuous energy bands also enhances the output power of the cell [42]. In this study, we examine the effect of the noise-induced quantum coherence on the performance of IB solar cells. We propose a model of an IBSC that absorbs continuous spectrums of sunlight instead of absorbing single wavelengths and investigate the Fano interference effects that arise from the absorption and emission of the incoherent solar light and the thermal phonons.

\section{NOISE-INDUCED QUANTUM COHERENCES IN $\Lambda$-TYPE SYSTEM DRIVEN BY INCOHERENT LIGHT}

In this paper, the $\Lambda$-type three-level system is used as a minimal building block for generating noise-induced coherence based on Fano interference [32], [43] in the proposed model for the IBSCs, so, in this section, we present a numerical study of the dynamical properties of such a three-level system. Our analysis is based on a fully microscopic quantum master equation of

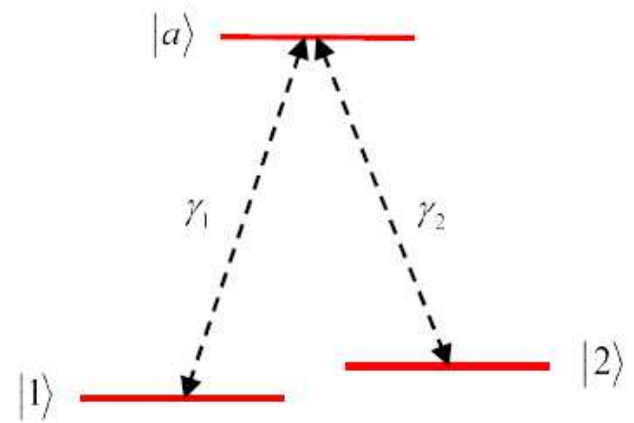

Fig. 1. Energy level scheme of a three-level $\Lambda$-type atomic system. $\gamma_{1}$ and $\gamma_{2}$ are decay rates.

density matrix elements (state populations and coherence) [44]. We consider a $\Lambda$-type three-level system shown in Fig. 1, where the upper state $|a\rangle$ is connected to two lower closely spaced states $|1\rangle$ and $|2\rangle$ by dipole-allowed transitions, this system interacts with incoherent light that is represented by a harmonic bath. The upper state decays with rate $\gamma_{1}$ and $\gamma_{2}$ through two different paths to lower states. We have derived the quantum motion equations of the density-matrix elements for this atomic system in the appendix, these equations that reveal coupling between populations and coherence due to noise are as follows:

$$
\dot{\rho}_{11}=\gamma_{1}\left[\left(\bar{n}_{1}+1\right) \rho_{a a}-\bar{n}_{1} \rho_{11}\right]-\frac{p \sqrt{\gamma_{1} \gamma_{2}}}{2} \bar{n}_{2}\left[\rho_{21}+\rho_{12}\right]
$$




$$
\begin{gathered}
\dot{\rho}_{22}=\gamma_{2}\left[\left(\bar{n}_{2}+1\right) \rho_{a a}-\bar{n}_{2} \rho_{22}\right]-\frac{p \sqrt{\gamma_{1} \gamma_{2}}}{2} \bar{n}_{1}\left[\rho_{12}+\rho_{21}\right] \\
\dot{\rho}_{12}=\left[-\frac{1}{2}\left(\gamma_{1} \bar{n}_{1}+\gamma_{2} \bar{n}_{2}\right)+i \Delta-\gamma_{d}\right] \rho_{12}+\frac{p \sqrt{\gamma_{1} \gamma_{2}}}{2}\left[\left(\bar{n}_{1}+\bar{n}_{2}+2\right) \rho_{a a}-\left(\overline{\mathrm{n}}_{1} \rho_{11}+\overline{\mathrm{n}}_{2} \rho_{22}\right)\right]
\end{gathered}
$$

where $\bar{n}_{1}$ and $\bar{n}_{2}$ are the photon average occupation numbers, $\gamma_{d}$ is the decoherence rate, $\Delta=\omega_{1}-\omega_{2}$ is the splitting of the levels $|1\rangle$ and $|2\rangle, p \sqrt{\gamma_{1} \gamma_{2}}$ is the cross-coupling that describe the effect of interference in decay paths from $|a\rangle$ to lower levels, and $p=\frac{\wp_{1 a} \cdot \wp_{2 a}}{\left|\wp_{1 a}\right|\left|\wp_{2 a}\right|}$ quantifies the angle between the $1 \rightarrow a$ and $2 \rightarrow a$ transition dipole moments (hereafter we assume $|p|=1$ ). Consider incoherent excitation of the system initially in the ground states $\left(\rho_{11}(0)=\rho_{22}(0)=0.5, \rho_{12}(0)=0\right)$ by isotropic and incoherent radiation (e.g., sunlight), to evaluate the dynamical behavior of the system, we obtain the time response of (1)-(3) with the assumption $\gamma_{1}=\gamma_{2}=\gamma, \gamma_{d}=0$ (for the moment we neglect decoherence, in the following we discuss its impact). We examine exact numerical responses based on the parameter $\Delta / \gamma$, which is the ratio of the ground level splitting to the decay width. First, Consider the regime of large splitting $\Delta$ between the ground state energy levels, where $\Delta / \gamma \square 1$ applicable to weakfield $\left(\bar{n}_{1}, \bar{n}_{2} \square 1\right)$ incoherent excitation [45], [46] of small to medium-sized molecules. Fig. 2 shows the exact time evolution $\operatorname{Re}\left[\rho_{12}(t)\right]$ obtained by numerical integration of (1)-(3) for $\gamma=1 \mathrm{GHz}, \Delta / \gamma=40$ and $\hbar \omega_{1}=1.25 \mathrm{eV}$. The coherence exhibits damped oscillations with frequency set by the product of $\Delta \cdot \gamma$ and decays with a time constant proportional to $1 / \gamma$ and the inverse of $\bar{n}$ and a maximum of $\operatorname{Re}\left[\rho_{12}(t)\right]$ increases with decreasing $\Delta$ and increasing $\bar{n}$ while its steady state maximum decreases. Note that no coherent driving fields are present and that these oscillations arise due to the sudden turn-on of the interaction with the incoherent radiation field, hence the name noise-induced coherence [30], [35].

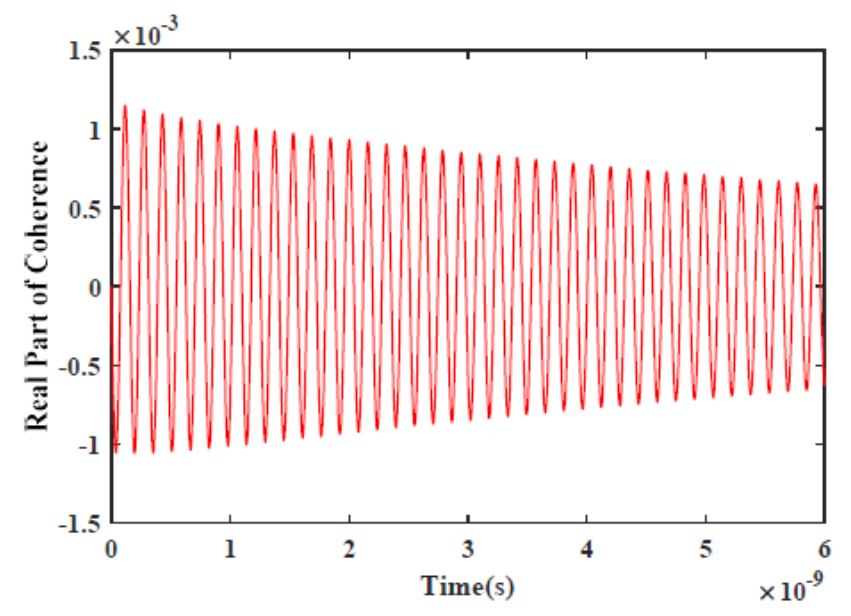

Fig. 2. The real part of the coherence $\rho_{12}^{R}(t)$. Result obtained for

$$
\Delta / \gamma=40, \gamma=1 \mathrm{GHz}, T=5800^{\circ} \mathrm{K} \text { and } \hbar \omega_{1}=1.25 \mathrm{eV} .
$$

In the opposite regime, $\Delta / \gamma \square 1$ which applies to incoherent excitation of a $\Lambda$-type system with very closely spaced ground levels of large-sized molecules [47], we find the absolute value of the coherence in the weak field $\operatorname{limit}\left(\bar{n}_{1}, \bar{n}_{2} \square 1\right)$. The time dependence of the coherence between two closely spaced ground state energy levels is shown in a log plot in Fig. 3 as a function 
of time for $\Delta / \gamma=0.024$, The most significant feature in Fig. 3 is the long lifetime of the coherence, for the given parameters, it survives for as long as $1 \mu \mathrm{s}$, more than $10^{3}$ times longer than the excited state radiative lifetime. In the limit of $\Delta \rightarrow 0$, the duration of the existence of the coherence approaches infinity, this reveals the crucial role of the ground state level splitting $\Delta$ in determining the noise-induced coherence dynamics. Maximizing the coherence lifetime, for example, is useful in designing quantum heat engines based on Fano interference [30], [32]. And our analysis suggests the benefits of using as small as possible $\Delta$. In the above analysis, we did not consider the effect of decoherence, however, in general, the $\Lambda$ - type three-level system is

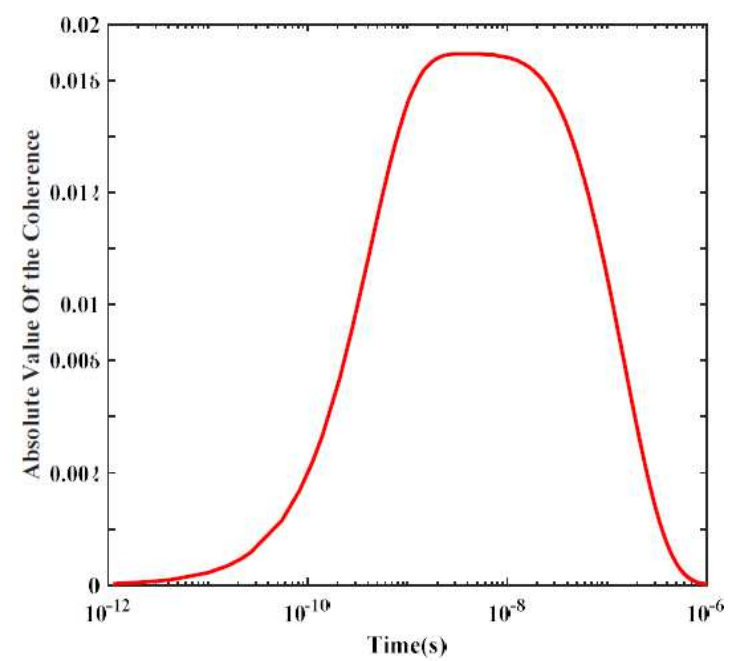

Fig. 3. The absolute value of the coherence $\left|\rho_{12}(t)\right|$ versus time for the $\Lambda$ -

type system in the limit of $\Delta / \gamma \square 1(\Delta / \gamma=0.024, \gamma=1 G H z)$.

exposed to the interaction with the environment, so this effect must be considered, and we model this effect by including the decoherence rate term $\gamma_{d}$ in the equations of motion[30]. As shown, long lifetime coherence is created in the regime of $\Delta / \gamma \square 1$

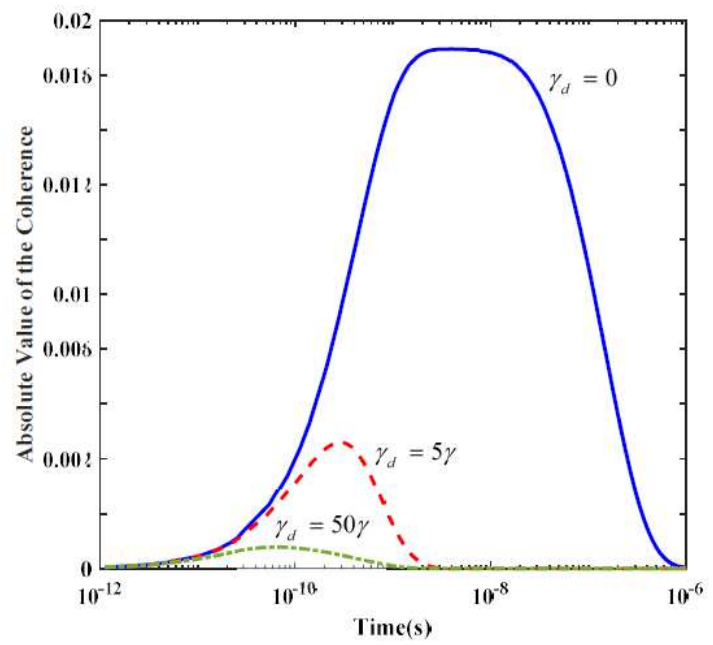

Fig. 4. The effect of environmental decoherence on the absolute value of the

$$
\begin{gathered}
\text { coherence for the } \Delta / \gamma=0.024 \text {. The imaginary part of the coherence is } \\
\text { negligible. }
\end{gathered}
$$


Therefore we examine the effect of decoherence in this regime. Fig. 4 illustrates the effects of decoherence on coherence for the $\Delta / \gamma=0.024$. We observe that decoherence lead to a suppression of the coherence, and cause a decrease in the amount of coherence. In addition, the time at which the coherence is disappeared decreases with increasing $\gamma_{d}$, suggesting that in the presence of decoherence, the dynamics are governed by a timescale $\tau_{d}=1 / \gamma_{d}$ that is shorter than that for spontaneous emission.

\section{THE PROPOSED MODELS FOR INTERMEDIATE BAND SOLAR CELLS}

In this part, we propose models of intermediate band solar cells to investigate the effect of quantum coherence in their performance. First, we present a six-level model of the intermediate band solar cell with two closely spaced ground levels and study the effect of coherence between these levels in the output power of the cell. This model can absorb only a few single wavelengths from the sunlight spectrum; therefore, we present a model that can absorb continuous spectra of sunlight. The density matrix method is used for these studies, and the required parameters will be obtained by the exact numerical solution.

\section{A. IBSC model with lower levels Fano interference}

The model presented to the intermediate band solar cell has six energy levels, as shown in Fig. 5. This structure consists of an energy level $|a\rangle$ in the conduction band, an intermediate level $|m\rangle$, and two lower levels $\left|b_{1}\right\rangle$ and $\left|b_{2}\right\rangle$ in the valence band and the levels $|c\rangle$ and $|v\rangle$ for

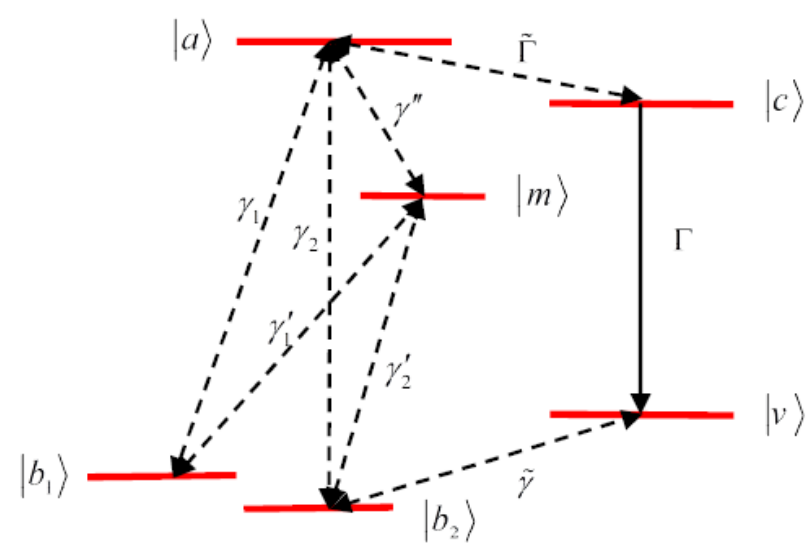
connecting to a load.

Fig. 5. Energy level diagram of an IB solar cell with the lower energy doublet. Solar radiation drives transitions between the levels $a, m$ and the two lower levels $b_{1}$ and $b_{2}$. Transitions $b_{1} \rightarrow v, b_{2} \rightarrow v$ and $a \rightarrow c$ are driven by ambient thermal phonons. Levels $c$ and $v$ are connected to a load.

We assume that this cell is exposed to sunlight radiation that contains photons with energies corresponding to the energy gaps $E_{a}-E_{m}, E_{m}-E_{b_{1}, b_{2}}$ and the band gap energy, $E_{a}-E_{b}$, and such photons are absorbed. In this model, like the one IB solar cells, photons are absorbed in a two-step process. Also, photon absorption is done in two channels. In one of the pathways, first the photon with energy $E_{m}-E_{b_{1}}$ is absorbed and an electron is promoted from the valence band level $b_{1}$ to the intermediate level $m$, then photon with energy $E_{a}-E_{m}$ promotes the electron from level $m$ to the conduction band level $a$. The same process of two-photon excitation can be carried out via $b_{2} \rightarrow m \rightarrow a$ transition channel. When the electron is transferred to the conduction level $a$, it can either pass through the load and contribute to the current and produce power or through the intermediate level go back to the valence band again and emit photons by the process of radiative recombination. As shown in 
Fig. 5, our proposed model includes three $\Lambda$-type structures $b_{1}-v-b_{2}, b_{1}-m-b_{2}$ and $b_{1}-a-b_{2}$. And as shown in section II, in a $\Lambda$ - type three-level system with two lower closely spaced states that is in interaction with the incoherent light, interference among different excitation/relaxation pathways can create coherence between the two lower levels. Therefore, in this model, multiple pathways of absorption and emission lead to inducing quantum coherence. We demonstrate that such coherence can cause an increase in photon absorption and therefore increases the power delivered to the load.

The interaction picture Hamiltonian in the rotating-wave approximation for this system is given by:

$$
\begin{aligned}
\hat{\mathrm{V}}(t)= & \ddot{\mathrm{y}} \sum_{\mathbf{k}}\left(g_{1 \mathbf{k}}|a\rangle\left\langle 1\left|e^{\mathrm{i} \omega_{1 a} t}+g_{2 \mathbf{k}}\right| a\right\rangle\langle 2| e^{\mathrm{i} \omega_{2 a} t}\right) \hat{a}_{\mathbf{k}} e^{-\mathrm{i} \nu_{k} t}+\ddot{\mathrm{y}} \sum_{\mathbf{q}}\left(\tilde{g}_{1 \mathbf{q}}|m\rangle\left\langle 1\left|e^{\mathrm{i} \omega_{1 m} t}+\tilde{g}_{2 \mathbf{q}}\right| m\right\rangle\langle 2| e^{\mathrm{i} \omega_{2 m} t}\right) \hat{a}_{\mathbf{q}} e^{-\mathrm{i} v_{q} t}+ \\
& \ddot{\mathrm{y}} \sum_{\mathbf{p}}\left(G_{1 \mathbf{p}}|v\rangle\left\langle 1\left|e^{\mathrm{i} \omega_{1 v} t}+G_{2 \mathbf{p}}\right| v\right\rangle\langle 2| e^{\mathrm{i} \omega_{2 v} t}\right) \hat{b}_{\mathbf{p}} e^{-\mathrm{i} v_{p} t}+\hbar \sum_{\mathbf{p}} \tilde{G}_{\mathbf{p}} \hat{b}_{\mathbf{p}} e^{\mathrm{i}\left(\omega_{a c}-v_{p}\right) t}|a\rangle\langle c|+\text { H.C. }
\end{aligned}
$$

where $\hat{a}_{\mathbf{k}}$ are radiation field operators and $\hat{b}_{\mathbf{p}}$ are thermal phonon operators and $g_{\mathbf{k}}, \tilde{g}_{\mathbf{q}}, G_{\mathbf{p}}$ and $\tilde{G}_{\mathbf{p}}$ are the coupling constant for the transition $b_{i} \leftrightarrow a, b_{i} \leftrightarrow m, b_{i} \leftrightarrow v$ and $c \leftrightarrow a$ (for $i=1,2$ ), respectively.

By following the general method used in the appendix, the density matrix equations are obtained as follows:

$$
\begin{gathered}
\dot{\rho}_{11}=\tilde{\gamma}_{1}\left[\left(1+n_{v}\right) \rho_{v v}-n_{v} \rho_{11}\right]+\gamma_{1}\left[(1+n) \rho_{a a}-n \rho_{11}\right]+\gamma_{1}^{\prime}\left[\left(1+n^{\prime}\right) \rho_{m m}-n^{\prime} \rho_{11}\right]-\left(\tilde{\gamma}_{12} n_{v}+\gamma_{12} n+\gamma_{12}^{\prime} n^{\prime}\right) \operatorname{Re}\left[\rho_{12}\right] \\
\dot{\rho}_{22}=\tilde{\gamma}_{2}\left[\left(1+n_{v}\right) \rho_{v v}-n_{v} \rho_{22}\right]+\gamma_{2}\left[(1+n) \rho_{a a}-n \rho_{22}\right]+\gamma_{2}^{\prime}\left[\left(1+n^{\prime}\right) \rho_{m m}-n^{\prime} \rho_{22}\right]-\left(\tilde{\gamma}_{12} n_{v}+\gamma_{12} n+\gamma_{12}^{\prime} n^{\prime}\right) \operatorname{Re}\left[\rho_{12}\right] \\
\dot{\rho}_{12}=\left(i \Delta-\gamma_{d}-\frac{1}{2}\left[\left(\gamma_{1}+\gamma_{2}\right) n+\left(\tilde{\gamma}_{1}+\tilde{\gamma}_{2}\right) n_{v}+\left(\gamma_{1}^{\prime}+\gamma_{2}^{\prime}\right) n^{\prime}\right]\right) \rho_{12}+\gamma_{12}\left[(n+1) \rho_{a a}-\frac{1}{2} n\left(\rho_{11}+\rho_{22}\right)\right]+ \\
\tilde{\gamma}_{12}\left[\left(n_{v}+1\right) \rho_{v v}-\frac{1}{2} n_{v}\left(\rho_{11}+\rho_{22}\right)\right]+\gamma_{12}^{\prime}\left[\left(n^{\prime}+1\right) \rho_{m m}-\frac{1}{2} n^{\prime}\left(\rho_{11}+\rho_{22}\right)\right] \\
\dot{\rho}_{c c}=\tilde{\Gamma}\left(1+n_{c}\right) \rho_{a a}-\left(\tilde{\Gamma} n_{c}+\Gamma\right) \rho_{c c} \\
\dot{\rho}_{m m}=\gamma_{1}^{\prime}\left[n^{\prime} \rho_{11}-\left(1+n^{\prime}\right) \rho_{m m}\right]+\gamma_{2}^{\prime}\left[n^{\prime} \rho_{22}-\left(1+n^{\prime}\right) \rho_{m m}\right]+\gamma^{\prime \prime}\left[\left(1+n^{\prime \prime}\right) \rho_{a a}-n^{\prime \prime} \rho_{m m}\right]+2 \gamma_{12}^{\prime} n^{\prime} \operatorname{Re}\left[\rho_{12}\right] \\
\rho_{11}+\rho_{22}+\rho_{a a}+\rho_{c c}+\rho_{v v}+\rho_{m m}=1
\end{gathered}
$$

where $n, n^{\prime}$ and $n^{\prime \prime}$ are the average occupation number of photons that drive $b_{i} \leftrightarrow a, b_{i} \leftrightarrow m$ and $m \leftrightarrow a$ transitions and given by:

$$
n=\frac{1}{\exp \left(\frac{E_{a b}}{k_{B} T_{S}}\right)-1}, n^{\prime}=\frac{1}{\exp \left(\frac{E_{m}-E_{b}}{k_{B} T_{s}}\right)-1} \text { and } n^{\prime \prime}=\frac{1}{\exp \left(\frac{E_{a}-E_{m}}{k_{B} T_{s}}\right)-1}
$$

$n_{c}$ and $n_{\nu}$ are the average occupation number of thermal phonons at ambient temperature $T_{a}$ that drive the $a \leftrightarrow c$ and $v \leftrightarrow b_{i}$ transitions, The average occupation numbers of these phonons are calculated by the following relationships: 


$$
n_{v}=\frac{1}{\exp \left(\frac{E_{v b}}{k_{B} T_{a}}\right)-1}, n_{c}=\frac{1}{\exp \left(\frac{E_{a c}}{k_{B} T_{a}}\right)-1} .
$$

In (5)-(11) $\gamma_{i}, \gamma_{i}^{\prime}, \gamma^{\prime \prime}, \tilde{\gamma}_{i}$ and $\tilde{\Gamma}$ are the spontaneous decay rates of the transitions $a \leftrightarrow b_{i}, m \leftrightarrow b_{i}, a \leftrightarrow m, v \leftrightarrow b_{i}$ and $a \leftrightarrow c$ (for $i=1,2$ ) respectively, $\gamma_{d}$ is the decoherence rate, $\Delta=\omega_{1}-\omega_{2}$ is the spacing between levels $b_{1}$ and $b_{2}$, also $\gamma_{12}, \gamma_{12}^{\prime}$ and $\tilde{\gamma}_{12}$ indicate the coherence between levels $b_{1}$ and $b_{2}$ that induced by interference in decay paths from $a, m$ and $v$ to lower levels respectively and are determined from the following relationships:

$$
\gamma_{12}=p \sqrt{\gamma_{1} \gamma_{2}}, \gamma_{12}^{\prime}=p^{\prime} \sqrt{\gamma_{1}^{\prime} \gamma_{2}^{\prime}} \text { and } \tilde{\gamma}_{12}=\tilde{p} \sqrt{\tilde{\gamma}_{1} \tilde{\gamma}_{2}}
$$

where $p, p^{\prime}$ and $\tilde{p}$ are the alignment factors of the dipole matrix elements that quantify the angle between the corresponding transition dipole moments, and are defined by the following relationships:

$$
p=\frac{\wp_{1 a} \cdot \wp_{2 a}}{\left|\wp_{1 a}\right|\left|\wp_{2 a}\right|}, p^{\prime}=\frac{\wp_{1 m} \cdot \wp_{2 m}}{\left|\wp_{1 m}\right|\left|\wp_{2 m}\right|} \text { and } \tilde{p}=\frac{\wp_{1 v} \cdot \wp_{2 v}}{\left|\wp_{1 v}\right|\left|\wp_{2 v}\right|}
$$

For maximum Fano interference, the angle between the dipole moments must be zero or 180 degrees, or in other words, $|p|=\left|p^{\prime}\right|=|\tilde{p}|=1$. Whereas, if the dipole moments are perpendicular to each other, i.e. $|p|=\left|p^{\prime}\right|=|\tilde{p}|=0$, there is no interference. In the scheme of Fig. 5, we assume that the load is connected to the levels $|c\rangle$ and $|v\rangle$, to absorb the produced power of the cell. The load is modeled by the decay rate $\Gamma$, so that $\Gamma=0$ corresponds to the open-circuit regime, whereas large $\Gamma$ is the short circuit limit.

\section{B. The IB solar cell model with mini bands}

The IBSC models discussed in the preceding section can absorb only three single wavelengths of sunlight spectrum. In order to absorb continuous spectra of sunlight, the energy levels should be replaced with energy bands. On the other hand, it has been shown that the quantum coherence can increase the output power of the solar cell structures that have a mini-band in their conduction band [42]. Therefore, in this section, we present a model of an IB solar cell that absorbs continuous spectrums of sunlight and consider the quantum coherence effects produced by Fano coupling associated with the emission and absorption mechanisms of the solar photons and the thermal phonons. The IBSC model is shown in Fig. 6. This model consists of mini

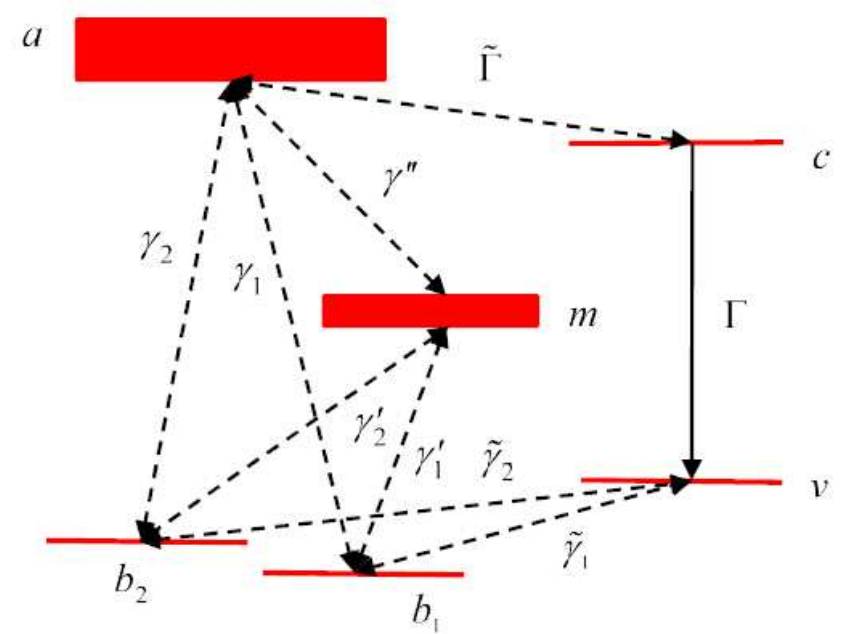

Fig. 6. Energy levels diagram for the IB solar cell model with mini bands. 
bands in the conduction and intermediate band, which we take the width of these bands $\Delta E$ and $\Delta E^{\prime}$ respectively. The other levels of this structure are similar to the model shown in Figure 5. In this model, the transition of electrons between level $|c\rangle$ and mini band $a$, as well as between levels $\left|b_{1}\right\rangle,\left|b_{2}\right\rangle$ and $|v\rangle$, is accomplished by the interaction of the system with the phonon reservoir. The electron transition between the ground levels and the mini bands, as well as between the mini bands itself, is carried out by the interaction of the system with the radiation field. We assume that solar photons with energies in the range $\left(E_{i a_{b}}, E_{i a_{b}}+\Delta E\right),\left(E_{i m_{b}}, E_{i m_{b}}+\Delta E^{\prime}\right)$ and $\left(E_{a_{b}}-E_{m_{b}}-\Delta E^{\prime}, E_{a_{b}}-E_{m_{b}}+\Delta E\right)$ where $a_{b}$ and $m_{b}$ are the bottom edge of the band $a$ and $m$, do transfers $\mathrm{a} \leftrightarrow b_{i}, m \leftrightarrow b_{i}$ and $m \leftrightarrow a$ (for $i=1,2$ ) respectively. The density matrix equations can be obtained from the following the method of [36] and a general formalism outlined in the appendix. In the present model, they read:

$$
\begin{aligned}
& \dot{\rho}_{11}= \tilde{\gamma}_{1}\left[\left(1+n_{1 v}\right) \rho_{v v}-n_{1 v} \rho_{11}\right]+\gamma_{1}^{\prime}\left[\left(1+n_{1 b}^{\prime}\right) \rho_{m_{b} m_{b}}-\int d m^{\prime} n_{1}^{\prime}\left(m^{\prime}\right) \rho_{11}\right]+\gamma_{1}\left[\left(1+n_{1 b}\right) \rho_{a_{b} a_{b}}-\int d a^{\prime} n_{1}\left(a^{\prime}\right) \rho_{11}\right]- \\
&\left(\gamma_{12} n_{2 b}+\tilde{\gamma}_{12} n_{2 v}+\gamma_{12}^{\prime} \mathrm{n}_{2 b}^{\prime}\right) \operatorname{Re}\left[\rho_{12}\right] \\
& \dot{\rho}_{22}= \tilde{\gamma}_{2}\left[\left(1+n_{2 v}\right) \rho_{v v}-n_{2 v} \rho_{22}\right]+\gamma_{2}^{\prime}\left[\left(1+n_{2 b}^{\prime}\right) \rho_{m_{b} m_{b}}-\int d m^{\prime} n_{2}^{\prime}\left(m^{\prime}\right) \rho_{22}\right]+\gamma_{2}\left[\left(1+n_{2 b}\right) \rho_{a_{b} a_{b}}-\int d a^{\prime} n_{2}\left(a^{\prime}\right) \rho_{22}\right]- \\
&\left(\gamma_{12} n_{1 b}+\tilde{\gamma}_{12} n_{1 v}+\gamma_{12}^{\prime} \mathrm{n}_{1 b}^{\prime}\right) \operatorname{Re}\left[\rho_{12}\right] \\
& \dot{\rho}_{12}=\left[\begin{array}{cl}
\left.i \Delta-1 / 2\left(\gamma_{1} n_{1 b}+\gamma_{2} n_{2 b}+\tilde{\gamma}_{1} n_{1 v}+\tilde{\gamma}_{2} n_{2 v}+\gamma_{1}^{\prime} n_{1 b}^{\prime}+\gamma_{2}^{\prime} n_{2 b}\right)\right] \rho_{12}+\frac{\gamma_{12}}{2}\left[\left(n_{1 b}+n_{2 b}+2\right) \rho_{a_{b} a_{b}}-\left(n_{1 b} \rho_{11}+n_{2 b} \rho_{22}\right)\right]+ \\
\frac{\tilde{\gamma}_{12}}{2}\left[\left(\mathrm{n}_{1 v}+n_{2 v}+2\right) \rho_{v v}-\left(\mathrm{n}_{1 v} \rho_{11}+n_{2 v} \rho_{22}\right)\right]+\frac{\gamma_{12}^{\prime}}{2}\left[\left(n_{1 b}^{\prime}+n_{2 b}^{\prime}+2\right) \rho_{m_{b} m_{b}}-\left(n_{1 b}^{\prime} \rho_{11}+n_{2 b}^{\prime} \rho_{22}\right)\right] \\
\dot{\rho}_{c c}=\tilde{\Gamma}\left[\left(1+n_{c b}\right) \rho_{a_{b} a_{b}}-\int d a^{\prime} n_{c}\left(a^{\prime}\right) \rho_{c c}\right]-\Gamma \rho_{c c} \\
\dot{\rho}_{v v}=\tilde{\gamma}_{1}\left[n_{1 v} \rho_{11}-\left(1+n_{1 v}\right) \rho_{v v}\right]+\tilde{\gamma}_{2}\left[n_{2 v} \rho_{22}-\left(1+n_{2 v}\right) \rho_{v v}\right]+\tilde{\gamma}_{12}\left(n_{1 v}+n_{2 v}\right) \operatorname{Re}\left[\rho_{12}\right]+\Gamma \rho_{c c} \\
\dot{\rho}_{a^{\prime} a^{\prime}}=\gamma_{1} n_{1}\left(a^{\prime}\right) \rho_{11}+\gamma_{2} n_{2}\left(a^{\prime}\right) \rho_{22}+\tilde{\Gamma} n_{c}\left(a^{\prime}\right) \rho_{c c}+\gamma^{\prime \prime} \int d m^{\prime} n_{a^{\prime}}^{\prime \prime}\left(m^{\prime}\right) \rho_{m^{\prime} m^{\prime}}-\gamma_{b}\left(n_{b}\left(a^{\prime}\right)+1\right) \rho_{a^{\prime} a^{\prime}} \\
\dot{\rho}_{m^{\prime} m^{\prime}}=\gamma_{1}^{\prime} n_{1}^{\prime}\left(m^{\prime}\right) \rho_{11}+\gamma_{2}^{\prime} n_{2}^{\prime}\left(m^{\prime}\right) \rho_{22}-\gamma^{\prime \prime} \int d a^{\prime} n_{m^{\prime}}^{\prime \prime}\left(a^{\prime}\right) \rho_{m^{\prime} m^{\prime}}-\gamma_{b}\left(n_{b}\left(m^{\prime}\right)+1\right) \rho_{m^{\prime} m^{\prime}} \\
\rho_{11}+\rho_{22}+\int d a^{\prime} \rho_{a^{\prime} a^{\prime}}+\int d m^{\prime} \rho_{m^{\prime} m^{\prime}}+\rho_{v v}+\rho_{c c}=1
\end{array}\right.
\end{aligned}
$$

Where $a^{\prime}$ and $m^{\prime}$ are levels inside mini bands $a$ and $m, n_{i}, n_{i}^{\prime}$ and $n^{\prime \prime}$ are the average occupation number of photons in transitions $\mathrm{a}^{\prime} \leftrightarrow b_{i}, m^{\prime} \leftrightarrow b_{i}$ and $m \leftrightarrow a$ (for $i=1,2$ ) respectively which are determined by the following relationships:

$$
n_{i}\left(a^{\prime}\right)=\frac{1}{\exp \left(\frac{E_{a^{\prime} b_{i}}}{k_{B} T_{S}}\right)-1}, n_{i}^{\prime}\left(m^{\prime}\right)=\frac{1}{\exp \left(\frac{E_{m^{\prime} b_{i}}}{k_{B} T_{S}}\right)-1} \text { and } n_{m^{\prime}}^{\prime \prime}\left(a^{\prime}\right)=\frac{1}{\exp \left(\frac{E_{a^{\prime} m^{\prime}}}{k_{B} T_{S}}\right)-1} \text {. }
$$

$n_{i v}$ and $n_{c}$ are the average occupation number of thermal phonons at ambient temperature $T_{a}$ that drive the $\mathrm{a}^{\prime} \leftrightarrow c$ and $v \leftrightarrow b_{i}$ transitions, the average occupation numbers of these phonons are calculated by the following relationships:

$$
n_{i v}=\frac{1}{\exp \left(\frac{E_{v b_{i}}}{k_{B} T_{a}}\right)-1} \text { and } n_{c}\left(a^{\prime}\right)=\frac{1}{\exp \left(\frac{E_{a^{\prime} c}}{k_{B} T_{a}}\right)-1}
$$


In obtaining the density matrix equations, we assume that the electrons transferred to the conduction and intermediate band fall to the bottom edge of these bands with the emission of phonon at the rate $\gamma_{b}$. The downward transitions from bands $a$ and $m$ occur exactly from the bottom edge of these bands, $a_{b}$ and $m_{b}$.

In (16)-(23) $\gamma_{i}, \gamma_{i}^{\prime}, \gamma^{\prime \prime}, \tilde{\gamma}_{i}$ and $\tilde{\Gamma}$ are the spontaneous decay rates of the corresponding transitions (see Fig. 6), and since the width of conduction and intermediate band is assumed to be small, we consider them constant, also $\gamma_{12}, \gamma_{12}^{\prime}$ and $\tilde{\gamma}_{12}$ indicate the coherence between levels $b_{1}$ and $b_{2}$ that induced by interference in decay paths from $a_{b}, m_{b}$ and $v$ to lower levels respectively and are obtained from (14) and (15). The rest of the used parameters are explained in Section III.A.

\section{SIMULATION RESULTS}

In this section, we investigate the effect of quantum coherence on the performance of both IBSC models presented in the previous section. For this purpose, the generated power and current-voltage characteristics of these models are calculated. We use the following relationships for calculating the voltage and current of solar cells in terms of the population of energy levels [40]:

$$
\begin{gathered}
e V=E_{c}-E_{v}+k_{B} T_{a} \ln \left(\frac{\rho_{c c}}{\rho_{v v}}\right) \\
j=e \Gamma \rho_{c c}
\end{gathered}
$$

where $E_{c}-E_{v}$ is the energy gap between the level $c$ and $v, \rho_{c c}$ and $\rho_{v v}$ are the populations of these levels respectively, $T_{a}$ is the ambient temperature and $\Gamma$ is the decay rate used to model the load. The power delivered to the load is calculated by $P=j \cdot V$. To calculate the levels population, we solve the density matrix equations describing each model in the steady state.

The exact analytical solution is almost impossible due to a large number of equations, therefore, we solve the equations numerically to obtain more accurate results. We first analyze the model presented in Fig. 5. In calculations, we take the decay rate $\gamma=1 \mathrm{GHz}$ and the band gap $E_{a}-E_{b}$ to be about $1.93 \mathrm{eV}$, in which the intermediate band solar cell efficiency is the highest theoretically [4]. We take the high and low bandgap energies to be $E_{m}-E_{b}=1.23 \mathrm{eV}$ and $E_{a}-E_{m}=0.7 \mathrm{eV}$ respectively. Also, in order to have maximum steady state coherence, according to the results of Section II, we assume that levels $b_{1}$ and $b_{2}$ are degenerate, $\Delta=0$. All parameters that used in the simulations are summarized in Table I. Figs. 7- 8 represent the current and output power produced by the described solar cell model as a function of voltage. The simulation results show that in the model shown in Fig. 5, when the intermediate level is closer to the conduction band the output current of the cell is higher. The reason is that in this case, the average photon number for transferring electrons from the intermediate level to the conduction band, is greater than that in which the intermediate level is closer to the valence band, therefore, more electrons are transferred from the intermediate level to the conduction band and participate in the creation of the current, but in the latter case, whereas the intermediate level due to the greater average number of photons is more populated, less electrons are transferred to the conduction band and the generated current decreases. In the structure of Fig. 5, the coherence between the levels 1 and 2 is generated by three interference channels, $b_{1} \leftrightarrow a \leftrightarrow b_{2}, b_{1} \leftrightarrow \nu \leftrightarrow b_{2}$ and $b_{1} \leftrightarrow m \leftrightarrow b_{2}$ which are shown with cross-coupling parameters $\gamma_{12}, \tilde{\gamma}_{12}$ and $\gamma_{12}^{\prime}$ respectively. The result of our calculations showed that the presence of interference in the channel $b_{1} \leftrightarrow a \leftrightarrow b_{2}$, which is shown by the parameter $\gamma_{12}$, reduces the coherence between the levels 1 and 2 and thus reduces the current through the load. Therefore, in our calculations, we assume that the coherence between the levels $b_{1}$ and $b_{2}$ is induced by the $\tilde{\gamma}_{12}$ and $\gamma_{12}^{\prime}$ parameters. Figure 7-a shows the induced coherence $\rho_{12}$ between $b_{1}$ and $b_{2}$, and Figure 7-b shows the current of three solar cell models with the same parameters as a function of voltage. The green dashed-line is for a single band gap solar cell, the red dash-dot line is for IB solar cell with a single ground level and the blue solid line is for IB solar cell with a 
TABLE I

PARAMETERS USED FOR SIMULATION OF THE MODEL PRESENTED IN FIG. 5.

\begin{tabular}{|c|c|c|}
\hline Parameter Name & Symbol & Value \\
\hline Solar Temperature & $T_{s}$ & $0.5 \mathrm{eV}$ \\
\hline Ambient Temperature & $T_{a}$ & $0.0259 \mathrm{eV}$ \\
\hline \multirow{7}{*}{$\begin{array}{l}\text { Energy gap between } \\
\text { levels }\end{array}$} & $E_{a b}=E_{a}-E_{b}$ & $1.93 \mathrm{eV}$ \\
\hline & $E_{c v}=E_{c}-E_{v}$ & $1.92 e V$ \\
\hline & $E_{a m}=E_{a}-E_{m}$ & $0.7 \mathrm{eV}$ \\
\hline & $E_{m b}=E_{m}-E_{b}$ & $1.23 \mathrm{eV}$ \\
\hline & $E_{a c}=E_{a}-E_{c}$ & $0.005 \mathrm{eV}$ \\
\hline & $E_{v b}=E_{v}-E_{b}$ & $0.005 e V$ \\
\hline & $\Delta=E_{12}=\hbar \omega_{12}$ & $0 e V$ \\
\hline \multirow{6}{*}{ Spontaneous decay rates } & $\gamma$ & $1 G H z$ \\
\hline & $\gamma_{1}=\gamma_{2}$ & $\gamma$ \\
\hline & $\gamma_{1}^{\prime}=\gamma_{2}^{\prime}=\gamma^{\prime}$ & $5 \gamma$ \\
\hline & $\gamma^{\prime \prime}$ & $10 \gamma$ \\
\hline & $\tilde{\gamma}_{1}=\tilde{\gamma}_{2}=\tilde{\gamma}$ & $100 \gamma$ \\
\hline & $\tilde{\Gamma}$ & $100 \gamma$ \\
\hline
\end{tabular}

(a) (b)

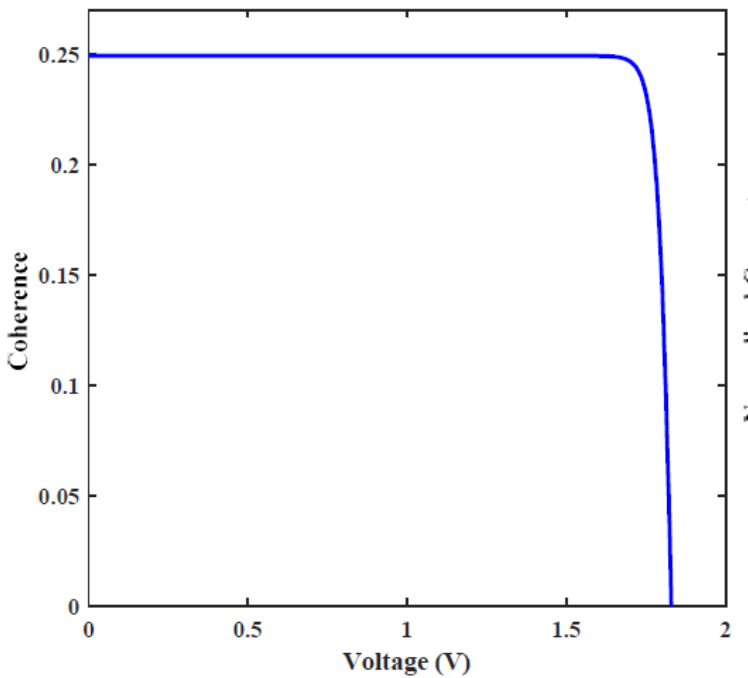

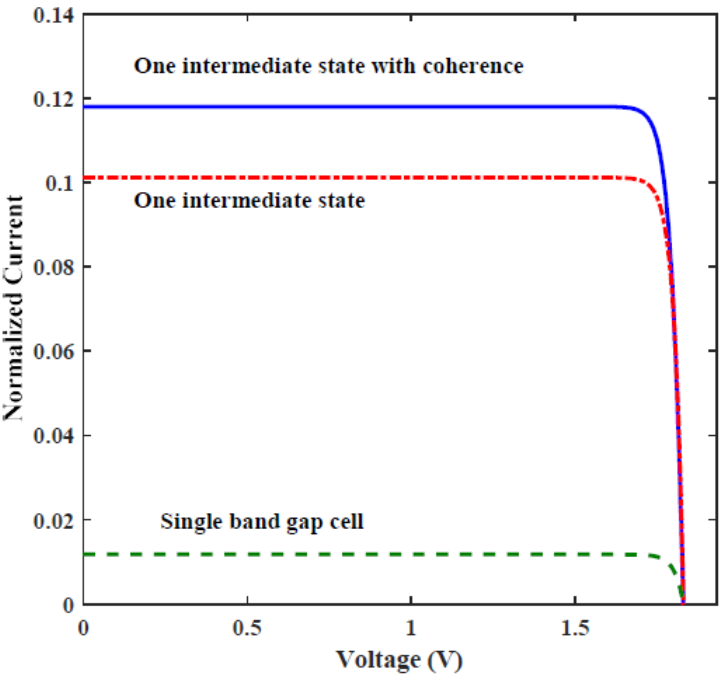

Fig. 7. (a) The coherence between the degenerate levels, $\rho_{12}$ as a function of the cell voltage. $\rho_{12}$ vanishes if $j=0$. (b) The normalized current $(j / e \gamma$ ) versus voltage characteristics of solar cell models presented in this section. 
doublet in the ground level (Fig. 5) in the presence of coherence. As can be seen, the presence of an intermediate level creates a large increase in the current of the cell. Also, the coherence enhances the current and consequently increases the produced power of the cell (Fig. 8). The coherence between two closely spaced ground levels causes the maximum output power of this model to be about $16 \%$ higher than the intermediate band solar cell with the single ground state.

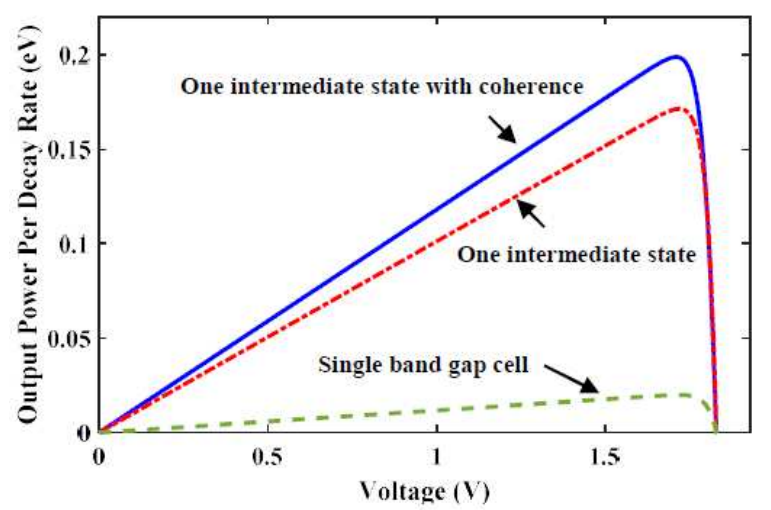

Fig. 8. The output power $(P / \gamma)$ generated by a solar cell as a function of voltage $V$ for three solar cell models.

Then, to investigate the coherence effect in the structure of Fig. 6, we solve the equations (16)-(23). Here, as in the previous case, in order to have maximum steady state coherence, we take two lower levels with zero spacing, $\Delta=0$. We consider the band gap energy from the bottom edge of the conduction band to the ground levels $E_{a_{b}}-E_{1,2}$ to be about $1.93 \mathrm{eV}$. Also, the simulation results show that in this model of the IBSC (Fig. 6) output current of the cell is higher when the IB is closer to the ground levels, so we take the high and low bandgap energies to be $E_{m_{b}}-E_{1,2}=0.7 \mathrm{eV}$ and $E_{a_{b}}-E_{m_{b}}=1.23 \mathrm{eV}$ respectively. We consider the widths of the bands $a$ and $m$ to be 100 and $10 \mathrm{meV}$, respectively. All parameters that used in the simulations are summarized in Table II. Here, as in the model presented in Fig. 5, the coherence generated by parameter $\gamma_{12}$, reduces the coherence between the levels 1 and 2 and thus reduces the current through the load. Therefore, in our calculations, we assume that the coherence between the levels $b_{1}$ and $b_{2}$ is induced by the $\tilde{\gamma}_{12}$ and $\gamma_{12}^{\prime}$ parameters. Figs. 9- 10 represent the current and output power of solar cell model as a function of voltage. The red dash-dot line is for IB solar cell with a single ground level and the blue solid line is for IB solar cell with two closely spaced ground levels with coherence between them.

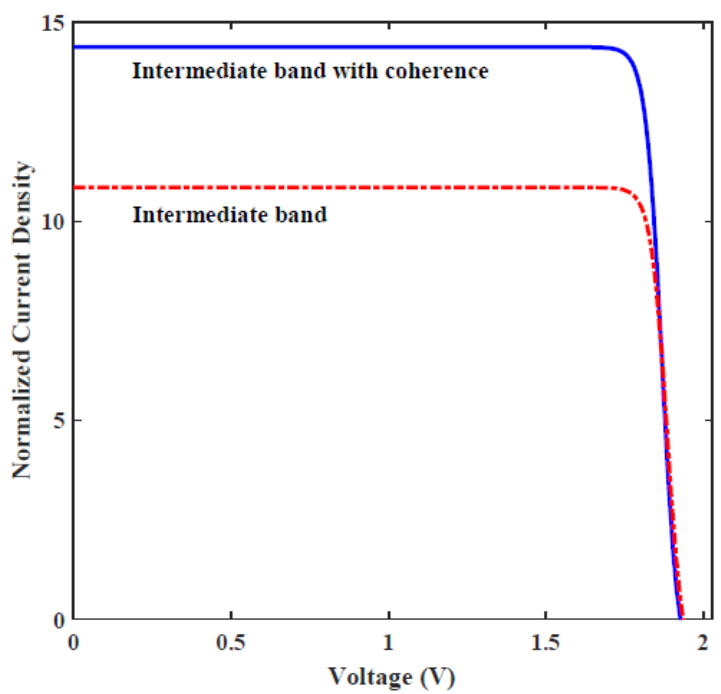

Fig. 9. The normalized current $(j / e \gamma)$ versus voltage characteristics of the IB solar cell model with mini-bands. The blue solid line is calculated in presence of coherence, and the red dash-dot line is for IB solar cell with a single ground level.
As seen in Figures, the inducing of coherence between closely

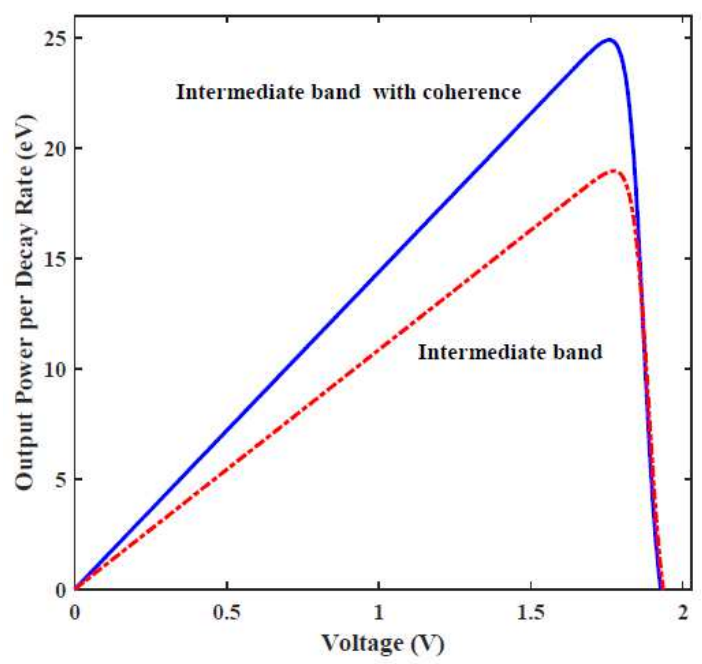

Fig. 10. The output power $(P / \gamma)$ of the IB solar cell model with minibands. The blue solid line is calculated in presence of coherence, and the red dash-dot line is for IB solar cell with a single ground level. 
spaced ground levels by incoherent mechanisms increases the generated current and the output power of the solar cell. The physical explanation for this increase is as follows: The absorption and emission mechanisms in channels $b_{1} \leftrightarrow v \leftrightarrow b_{2}$ and $b_{1} \leftrightarrow m_{b} \leftrightarrow b_{2}$ interfere with each other. This quantum interference

TABLE II

PARAMETERS USED FOR SIMULATION OF THE IB SOLAR CELL MODEL DEPICTED IN FIG. 6.

\begin{tabular}{|c|c|c|}
\hline Parameter Name & Symbol & Value \\
\hline Solar Temperature & $T_{s}$ & $0.5 \mathrm{eV}$ \\
\hline Ambient Temperature & $T_{a}$ & $0.0259 e V$ \\
\hline \multirow{7}{*}{$\begin{array}{l}\text { Energy gap between } \\
\text { levels }\end{array}$} & $E_{a 1}=E_{a_{b}}-E_{1}$ & $1.93 \mathrm{eV}$ \\
\hline & $E_{c v}=E_{c}-E_{v}$ & $1.92 \mathrm{eV}$ \\
\hline & $E_{a_{b} m_{b}}=E_{a_{b}}-E_{m_{b}}$ & $1.23 e V$ \\
\hline & $E_{m_{b} 1}=E_{m_{b}}-E_{1}$ & $0.7 \mathrm{eV}$ \\
\hline & $E_{a_{b} c}=E_{a_{b}}-E_{c}$ & $0.005 \mathrm{eV}$ \\
\hline & $E_{v b}=E_{v}-E_{b}$ & $0.005 \mathrm{eV}$ \\
\hline & $\Delta=E_{12}=\hbar \omega_{12}$ & $0 e V$ \\
\hline \multirow{10}{*}{ Spontaneous decay rates } & $\gamma$ & $1 G H z$ \\
\hline & $\gamma_{1}$ & $\gamma$ \\
\hline & $\gamma_{2}$ & $0.1 \gamma$ \\
\hline & $\gamma_{1}^{\prime}$ & $10 \gamma$ \\
\hline & $\gamma_{2}^{\prime}$ & $1.1 \gamma$ \\
\hline & $\gamma^{\prime \prime}$ & $5 \gamma$ \\
\hline & $\tilde{\gamma}_{1}$ & $50 \gamma$ \\
\hline & $\tilde{\gamma}_{2}$ & $5.5 \gamma$ \\
\hline & $\tilde{\Gamma}$ & $50 \gamma$ \\
\hline & $\gamma_{b}$ & $1000 \gamma$ \\
\hline \multirow{2}{*}{ Mini Bands Width } & $\Delta E$ & $100 m e V$ \\
\hline & $\Delta E^{\prime}$ & $10 \mathrm{meV}$ \\
\hline
\end{tabular}


induces coherence between the two ground levels $b_{1}$ and $b_{2}$, which is determined by parameters $\tilde{\gamma}_{12}=\tilde{p} \sqrt{\tilde{\gamma}_{1} \tilde{\gamma}_{2}}$ and $\gamma_{12}^{\prime}=p^{\prime} \sqrt{\gamma_{1}^{\prime} \gamma_{2}^{\prime}}$. This coherence has the maximum value when $|\tilde{p}|=\left|p^{\prime}\right|=1$. The induced coherence between the levels $b_{1}$ and $b_{2}$ causes the population displacement between these levels, so the level with the strongest coupling to the upper levels is more populated. The higher population in the energy state with stronger coupling increases the absorption of photons and leads to an enhancement of the current and thus increases the output power of the cell (solid line in Figs. 9 - 10). Fig. 10 shows that the maximum output power of the intermediate band solar cell depicted in Fig. 6 in the case of inducing coherence between two ground levels is about $31 \%$ higher than the model that has only one ground level. Although it should be noted that, according to the results of the calculations, increasing the width of the IB reduces the coherence effect in the enhancement of the current and output power of the solar cell. However, by expanding the width of the conduction band and despite decreasing the output power due to the enhancement of thermalization loss, the effect of quantum coherence in increasing the solar cell power is still observed

\section{CONCLUSIONS}

Due to the limited absorption coefficient in quantum dot based IB solar, we consider an atomic-like model of QDs-IB solar cells and it was shown that quantum coherence can increase the solar cell output power by increasing more photon absorption. In the presented models, coherence is generated between two ground energy levels due to interference between emission and absorption pathways. Such coherence can enhance photon absorption and suppress unwanted emission yielding increase of the photocurrent. We compare different models of the single-wavelength solar cell, including single-junction cell, single intermediate state and IB cell with ground state doublet, and we observed the effects of intermediate state and quantum coherence in increasing the produced power of solar cells. Finally, we discussed the effects of quantum coherence when the intermediate and conduction energy levels were considered as energy bands.

\section{APPENDIX}

\section{DERIVATION OF DENSITY MATRIX EQUATIONS}

Here we derive equations of motion for the density matrix elements with Fano interference terms. For simplicity we consider a $\Lambda$-type three-level atomic system shown in Fig. 1, where the upper state $|a\rangle$ is connected to two lower closely spaced states $|1\rangle$ and $|2\rangle$ by the dipole-allowed transition. We define the transition frequencies as $\omega_{1}$ and $\omega_{2}$, such that $E_{a 1}=E_{a}-E_{1}=\hbar \omega_{1}$ and $E_{a 2}=E_{a}-E_{2}=\hbar \omega_{2}$. The interaction picture Hamiltonian in the rotating-wave approximation for this system coupled to a harmonic bath is:

$$
\hat{\mathrm{V}}(t)=\ddot{\mathrm{y}} \sum_{k} g_{1 k} \hat{a}_{k} e^{i\left(\omega_{1}-v_{k}\right) t}|a\rangle\left\langle 1\left|+\ddot{\mathrm{y}} \sum_{q} g_{2 q} \hat{a}_{q} e^{i\left(\omega_{2}-v_{q}\right) t}\right| a\right\rangle\langle 2|+
$$

where $\hat{a}_{k}\left(\hat{a}_{q}\right)$ are radiation field operators and $g_{1 k}\left(g_{2 q}\right)$ is the atom-photon coupling constants for transitions $a \leftrightarrow 1(a \leftrightarrow 2$

)and is given by:

$$
\mathrm{g}_{k}=-\frac{\wp_{b a} \cdot \hat{O}_{k} \mathrm{E}_{k}}{\hbar}
$$

We assume that system interacts with incoherent thermal reservoir described by the density operator $\hat{\rho}_{R}$. The equation of motion for the density operator of the three-level system is [44]:

$$
\frac{d \hat{\rho}(t)}{d t}=-\frac{i}{\hbar} \operatorname{Tr}_{R}\left[\hat{\mathrm{V}}(t), \hat{\rho}\left(t_{0}\right) \otimes \hat{\rho}_{R}\left(t_{0}\right)\right]-\frac{1}{\hbar^{2}} \operatorname{Tr}_{R} \int_{t_{0}}^{t}\left[\hat{\mathrm{V}}(t),\left[\hat{\mathrm{V}}\left(t^{\prime}\right), \hat{\rho}\left(t^{\prime}\right) \otimes \hat{\rho}_{R}\left(t_{0}\right)\right]\right] d t^{\prime}
$$


Inserting $\hat{\mathrm{V}}(t)$ into (30) and taking into account that for incoherent source $\left\langle\hat{a}_{k}\right\rangle=\left\langle\hat{a}_{k}^{\dagger}\right\rangle=0$ and $\left\langle\hat{a}_{k} \hat{a}_{k^{\prime}}\right\rangle=\left\langle\hat{a}_{k}^{\dagger} \hat{a}_{k^{\prime}}^{\dagger}\right\rangle=0$, where $\langle\hat{A}\rangle$ stands for $\operatorname{Tr}_{R}\left(\hat{\rho}_{R}\left(t_{0}\right) \hat{A}\right)$, we find :

$$
\begin{aligned}
& \frac{d \hat{\rho}(t)}{d t}=-\int_{t_{0}}^{t} d t^{\prime}\left\{\sum_{k, k^{\prime}} g_{1 k} g_{1 k^{\prime}} e^{i\left(\omega_{1}-v_{k}\right) t-i\left(\omega_{1}-v_{k^{\prime}}\right) t^{\prime}} \operatorname{Tr}_{R}\left[\hat{a}_{k}|a\rangle\langle 1|,\left[a_{k^{\prime}}^{\dagger}|1\rangle\langle a|, \hat{\rho}\left(t^{\prime}\right) \otimes \hat{\rho}_{R}\left(t_{0}\right)\right]\right]\right. \\
& +\sum_{k, q^{\prime}} g_{1 k} g_{2 q^{\prime}} e^{i\left(\omega_{1}-v_{k}\right) t-i\left(\omega_{2}-v_{q^{\prime}}\right) t^{\prime}} \operatorname{Tr}_{R}\left[\hat{a}_{k}|a\rangle\langle 1|,\left[a_{q^{\prime}}^{\dagger}|2\rangle\langle a|, \hat{\rho}\left(t^{\prime}\right) \otimes \hat{\rho}_{R}\left(t_{0}\right)\right]\right] \\
& +\sum_{q, \mathrm{k}^{\prime}} g_{2 q} g_{1 k^{\prime}} e^{i\left(\omega_{2}-v_{q}\right) t-i\left(\omega_{1}-v_{k^{\prime}}\right) t^{\prime}} \operatorname{Tr}_{R}\left[\hat{a}_{q}|a\rangle\langle 2|,\left[a_{k^{\prime}}^{\dagger}|1\rangle\langle a|, \hat{\rho}\left(t^{\prime}\right) \otimes \hat{\rho}_{R}\left(t_{0}\right)\right]\right] \\
& +\sum_{q, q^{\prime}} g_{2 q} g_{2 q^{\prime}} e^{i\left(\omega_{2}-v_{q}\right) t-i\left(\omega_{2}-v_{q^{\prime}}\right) t} \operatorname{Tr}_{R}\left[\hat{a}_{q}|a\rangle\langle 2|,\left[a_{q^{\prime}}^{\dagger}|2\rangle\langle a|, \hat{\rho}\left(t^{\prime}\right) \otimes \hat{\rho}_{R}\left(t_{0}\right)\right]\right] \\
& +\sum_{k, k^{\prime}} g_{1 k} g_{1 k^{\prime}} e^{-i\left(\omega_{1}-v_{k}\right) t+i\left(\omega_{1}-v_{k^{\prime}}\right) t} \operatorname{Tr}_{R}\left[\hat{a}_{k}^{\dagger}|1\rangle\langle a|,\left[a_{k^{\prime}}|a\rangle\langle 1|, \hat{\rho}\left(t^{\prime}\right) \otimes \hat{\rho}_{R}\left(t_{0}\right)\right]\right] \\
& +\sum_{k, q^{\prime}} g_{1 k} g_{2 q^{\prime}} e^{-i\left(\omega_{1}-v_{k}\right) t+i\left(\omega_{2}-v_{q^{\prime}}\right) t} \operatorname{Tr}_{R}\left[\hat{a}_{k}^{\dagger}|1\rangle\langle a|,\left[a_{q^{\prime}}|a\rangle\langle 2|, \hat{\rho}\left(t^{\prime}\right) \otimes \hat{\rho}_{R}\left(t_{0}\right)\right]\right] \\
& +\sum_{q, \mathrm{k}^{\prime}} g_{2 q} g_{1 k^{\prime}}, e^{-i\left(\omega_{2}-v_{q}\right) t+i\left(\omega_{1}-v_{k^{\prime}}\right) t} \operatorname{Tr}_{R}\left[\hat{a}_{q}^{\dagger}|2\rangle\langle a|,\left[a_{k^{\prime}}|a\rangle\langle 1|, \hat{\rho}\left(t^{\prime}\right) \otimes \hat{\rho}_{R}\left(t_{0}\right)\right]\right] \\
& \left.+\sum_{q, q^{\prime}} g_{2 q} g_{2 q^{\prime}} e^{-i\left(\omega_{2}-v_{q}\right) t+i\left(\omega_{2}-v_{q^{\prime}}\right) t^{\prime}} \operatorname{Tr}_{R}\left[\hat{a}_{q}^{\dagger}|2\rangle\langle a|,\left[a_{q^{\prime}}|a\rangle\langle 2|, \hat{\rho}\left(t^{\prime}\right) \otimes \hat{\rho}_{R}\left(t_{0}\right)\right]\right]\right\}
\end{aligned}
$$

Since trace is invariant under cycling permutation of the operators the terms with $\operatorname{Tr}_{R}$ can be written as:

$$
\begin{aligned}
& \operatorname{Tr}_{R}\left[\hat{a}_{k}|a\rangle\langle 1|,\left[a_{k^{\prime}}^{\dagger}|1\rangle\langle a|, \hat{\rho}\left(t^{\prime}\right) \otimes \hat{\rho}_{R}\left(t_{0}\right)\right]\right]=|a\rangle\left\langle a\left|\left[\left\langle\hat{a}_{k} \hat{a}_{k^{\prime}}^{\dagger}\right\rangle \rho-\left\langle\hat{a}_{k^{\prime}}^{\dagger} \hat{a}_{k}\right\rangle \rho_{11}\right]+\left[\left\langle\hat{a}_{k^{\prime}}^{\dagger} \hat{a}_{k}\right\rangle \rho-\left\langle\hat{a}_{k} \hat{a}_{k^{\prime}}^{\dagger}\right\rangle \rho_{a a}\right]\right| 1\right\rangle\langle 1| \\
& \operatorname{Tr}_{R}\left[\hat{a}_{k}|a\rangle\langle 1|,\left[a_{q^{\prime}}^{\dagger}|2\rangle\langle a|, \hat{\rho}\left(t^{\prime}\right) \otimes \hat{\rho}_{R}\left(t_{0}\right)\right]\right]=\left[\left\langle\hat{a}_{q^{\prime}}^{\dagger} \hat{a}_{k}\right\rangle \rho-\left\langle\hat{a}_{k} \hat{a}_{q^{\prime}}^{\dagger}\right\rangle \rho_{a a}\right]|2\rangle\left\langle 1\left|-\left\langle\hat{a}_{q^{\prime}}^{\dagger} \hat{a}_{k}\right\rangle \rho_{12}\right| a\right\rangle\langle a| \\
& \operatorname{Tr}_{R}\left[\hat{a}_{q}|a\rangle\langle 2|,\left[a_{k^{\prime}}^{\dagger}|1\rangle\langle a|, \hat{\rho}\left(t^{\prime}\right) \otimes \hat{\rho}_{R}\left(t_{0}\right)\right]\right]=\left[\left\langle\hat{a}_{k^{\prime}}^{\dagger} \hat{a}_{q}\right\rangle \rho-\left\langle\hat{a}_{q} \hat{a}_{k^{\prime}}^{\dagger}\right\rangle \rho_{a a}\right]|1\rangle\left\langle 2\left|-\left\langle\hat{a}_{k^{\prime}}^{\dagger} \hat{a}_{k}\right\rangle \rho_{21}\right| a\right\rangle\langle a| \\
& \operatorname{Tr}_{R}\left[\hat{a}_{q}|a\rangle\langle 2|,\left[a_{q^{\prime}}^{\dagger}|2\rangle\langle a|, \hat{\rho}\left(t^{\prime}\right) \otimes \hat{\rho}_{R}\left(t_{0}\right)\right]\right]=\left[\left\langle\hat{a}_{q^{\prime}}^{\dagger} \hat{a}_{q}\right\rangle \rho-\left\langle\hat{a}_{a^{\prime}} \hat{a}_{q^{\prime}}^{\dagger}\right\rangle \rho_{a a}\right]|2\rangle\langle 2|+| a\rangle\langle a|\left[\left\langle\hat{a}_{q} \hat{a}_{q^{\prime}}^{\dagger}\right\rangle \rho-\left\langle\hat{a}_{q^{\prime}}^{\dagger} \hat{a}_{q}\right\rangle \rho_{22}\right] \\
& \operatorname{Tr}_{R}\left[\hat{a}_{k}^{\dagger}|1\rangle\langle a|,\left[a_{k^{\prime}}|a\rangle\langle 1|, \hat{\rho}\left(t^{\prime}\right) \otimes \hat{\rho}_{R}\left(t_{0}\right)\right]\right]=|1\rangle\left\langle 1\left|\left[\left\langle\hat{a}_{k}^{\dagger} \hat{a}_{k^{\prime}}\right\rangle \rho-\left\langle\hat{a}_{k^{\prime}} \hat{a}_{k}^{\dagger}\right\rangle \rho_{a a}\right]+\left[\left\langle\hat{a}_{k^{\prime}} \hat{a}_{k}^{\dagger}\right\rangle \rho-\left\langle\hat{a}_{k}^{\dagger} \hat{a}_{k^{\prime}}\right\rangle \rho_{11}\right]\right| a\right\rangle\langle a| \\
& \operatorname{Tr}_{R}\left[\hat{a}_{k}^{\dagger}|1\rangle\langle a|,\left[a_{q^{\prime}}|a\rangle\langle 2|, \hat{\rho}\left(t^{\prime}\right) \otimes \hat{\rho}_{R}\left(t_{0}\right)\right]\right]=|1\rangle\left\langle 2\left|\left[\left\langle\hat{a}_{k}^{\dagger} \hat{a}_{q^{\prime}}\right\rangle \rho-\left\langle\hat{a}_{q^{\prime}} \hat{a}_{k}^{\dagger}\right\rangle \rho_{a a}\right]-\left\langle\hat{a}_{k}^{\dagger} \hat{a}_{q^{\prime}}\right\rangle \rho_{21}\right| a\right\rangle\langle a| \\
& \operatorname{Tr}_{R}\left[\hat{a}_{q}^{\dagger}|2\rangle\langle a|,\left[a_{k^{\prime}}|a\rangle\langle 1|, \hat{\rho}\left(t^{\prime}\right) \otimes \hat{\rho}_{R}\left(t_{0}\right)\right]\right]=|2\rangle\left\langle 1\left|\left[\left\langle\hat{a}_{q}^{\dagger} \hat{a}_{k^{\prime}}\right\rangle \rho-\left\langle\hat{a}_{k^{\prime}} \hat{a}_{q}^{\dagger}\right\rangle \rho_{a a}\right]-\left\langle\hat{a}_{q}^{\dagger} \hat{a}_{k^{\prime}}\right\rangle \rho_{12}\right| a\right\rangle\langle a| \\
& \operatorname{Tr}_{R}\left[\hat{a}_{q}^{\dagger}|2\rangle\langle a|,\left[a_{q^{\prime}}|a\rangle\langle 2|, \hat{\rho}\left(t^{\prime}\right) \otimes \hat{\rho}_{R}\left(t_{0}\right)\right]\right]=|2\rangle\left\langle 2\left|\left[\left\langle\hat{a}_{q}^{\dagger} \hat{a}_{q^{\prime}}\right\rangle \rho-\left\langle\hat{a}_{q^{\prime}} \hat{a}_{q}^{\dagger}\right\rangle \rho_{a a}\right]+\left[\left\langle\hat{a}_{q^{\prime}} \hat{a}_{q}^{\dagger}\right\rangle \rho-\left\langle\hat{a}_{q^{\dagger}}^{\dagger} \hat{a}_{q^{\prime}}\right\rangle \rho_{22}\right]\right| a\right\rangle\langle a|
\end{aligned}
$$

Next, we take into account $\left\langle\hat{a}_{k}^{\dagger} \hat{a}_{k^{\prime}}\right\rangle=\bar{n}_{k} \delta_{k k^{\prime}}$ and $\left\langle\hat{a}_{k} \hat{a}_{k^{\prime}}^{\dagger}\right\rangle=\left(\overline{\mathrm{n}}_{k}+1\right) \delta_{k k^{\prime}}$. The sum over $k$ can be replaced by an integral through the prescription $\sum_{k} \rightarrow \frac{V_{p h}}{\pi^{2}} \int_{0}^{\infty} d k k^{2}$, where $V_{p h}$ is the photon volume. In the present paper, we are interested in the steady-state values of the density matrix. Thus, one can assume that $\hat{\rho}\left(\mathrm{t}^{\prime}\right)$ is a slowly varying function of time and replace $\hat{\rho}\left(\mathrm{t}^{\prime}\right)$ with $\hat{\rho}(\mathrm{t})$ under the integral over $t^{\prime}$ (Markov approximation). Then we extend integration over $t^{\prime}$ to $\infty$ and use $\int_{t_{0}}^{\infty} d t^{\prime} e^{i\left(\omega-v_{k}\right)\left(t-t^{\prime}\right)}=\pi \delta\left(\omega-v_{k}\right)$. As a result, the evolution equation for the atomic density operator reduces to: 


$$
\begin{aligned}
& \frac{d \hat{\rho}(t)}{d t}=-\frac{V_{p h}}{c \pi}\left\{k_{1}^{2} g_{1 k_{1}}^{2}\left(|a\rangle\left\langle a\left|\left[\left(\overline{\mathrm{n}}_{k_{1}}+1\right) \hat{\rho}(\mathrm{t})-\bar{n}_{k_{1}} \rho_{11}(t)\right]+\left[\overline{\mathrm{n}}_{k_{1}} \hat{\rho}(\mathrm{t})-\left(\overline{\mathrm{n}}_{k_{1}}+1\right) \rho_{a a}(\mathrm{t})\right]\right| 1\right\rangle\langle 1|\right)+\right. \\
& \mathrm{k}_{2}^{2} g_{1 k_{2}} g_{2 k_{2}} e^{i\left(\omega_{1}-\omega_{2}\right) t}\left(\left[n_{k_{2}} \hat{\rho}(t)-\left(\mathrm{n}_{k_{2}}+1\right) \rho_{a a}(t)\right]|2\rangle\left\langle 1\left|-n_{k_{2}} \rho_{12}(t)\right| a\right\rangle\langle a|\right)+ \\
& \mathrm{k}_{1}^{2} g_{2 k_{1}} g_{1 k_{1}} e^{i\left(\omega_{2}-\omega_{1}\right) t}\left(\left[n_{k_{1}} \hat{\rho}(t)-\left(\mathrm{n}_{k_{1}}+1\right) \rho_{a a}(t)\right]|1\rangle\left\langle 2\left|-n_{k_{1}} \rho_{21}(t)\right| a\right\rangle\langle a|\right)+ \\
& k_{2}^{2} g_{2 k_{2}}^{2}\left(\left[\overline{\mathrm{n}}_{k_{2}} \hat{\rho}(\mathrm{t})-\left(\bar{n}_{k_{2}}+1\right) \rho_{a a}(t)\right]|2\rangle\langle 2|+| a\rangle\langle a|\left[\left(\overline{\mathrm{n}}_{k_{2}}+1\right) \hat{\rho}(\mathrm{t})-\overline{\mathrm{n}}_{k_{2}} \rho_{22}(\mathrm{t})\right]\right)+ \\
& k_{1}^{2} g_{1 k_{1}}^{2}\left(|1\rangle\left\langle 1\left|\left[\overline{\mathrm{n}}_{k_{1}} \hat{\rho}(\mathrm{t})-\left(\bar{n}_{k_{1}}+1\right) \rho_{a a}(t)\right]+\left[\left(\overline{\mathrm{n}}_{k_{1}}+1\right) \hat{\rho}(\mathrm{t})-\overline{\mathrm{n}}_{k_{1}} \rho_{11}(\mathrm{t})\right]\right| a\right\rangle\langle a|\right)+ \\
& \mathrm{k}_{2}^{2} g_{1 k_{2}} g_{2 k_{2}} e^{-i\left(\omega_{1}-\omega_{2}\right) t}\left(|1\rangle\left\langle 2\left|\left[n_{k_{2}} \hat{\rho}(t)-\left(\mathrm{n}_{k_{2}}+1\right) \rho_{a a}(t)\right]-n_{k_{2}} \rho_{21}(t)\right| a\right\rangle\langle a|\right)+ \\
& \mathrm{k}_{1}^{2} g_{2 k_{1}} g_{1 k_{1}} e^{-i\left(\omega_{2}-\omega_{1}\right) t}\left(|2\rangle\left\langle 1\left|\left[n_{k_{1}} \hat{\rho}(t)-\left(\mathrm{n}_{k_{1}}+1\right) \rho_{a a}(t)\right]-n_{k_{1}} \rho_{12}(t)\right| a\right\rangle\langle a|\right)+ \\
& \left.k_{2}^{2} g_{2 k_{2}}^{2}\left(|2\rangle\left\langle 2\left|\left[\overline{\mathrm{n}}_{k_{2}} \hat{\rho}(\mathrm{t})-\left(\bar{n}_{k_{2}}+1\right) \rho_{a a}(t)\right]+\left[\left(\overline{\mathrm{n}}_{k_{2}}+1\right) \hat{\rho}(\mathrm{t})-\overline{\mathrm{n}}_{k_{2}} \rho_{22}(\mathrm{t})\right]\right| a\right\rangle\langle a|\right)\right\}
\end{aligned}
$$

where $k_{1,2}=\omega_{1,2} / c$. We assume that levels 1 and 2 are close to each other. Then approximately, $k_{1} \approx k_{2} \approx k_{0}$, where $k_{0}=\left(\mathrm{k}_{1}+\mathrm{k}_{2}\right) / 2$. Introducing notations $\gamma_{1}=\frac{2 k_{0}^{2} V_{p h} g_{1 k_{0}}^{2}}{\pi c}, \quad \gamma_{2}=\frac{2 k_{0}^{2} V_{p h} g_{2 k_{0}}^{2}}{\pi c}$ one can rewrite (50) as:

$$
\begin{aligned}
& -2 \frac{d \hat{\rho}(t)}{d t}=\gamma_{1}\left(|a\rangle\left\langle a\left|\left[\left(\overline{\mathrm{n}}_{k_{1}}+1\right) \hat{\rho}(\mathrm{t})-\bar{n}_{k_{1}} \rho_{11}(t)\right]+\left[\overline{\mathrm{n}}_{k_{1}} \hat{\rho}(\mathrm{t})-\left(\overline{\mathrm{n}}_{k_{1}}+1\right) \rho_{a a}(\mathrm{t})\right]\right| 1\right\rangle\langle 1|\right)+ \\
& p \sqrt{\gamma_{1} \gamma_{2}} e^{i\left(\omega_{1}-\omega_{2}\right) t}\left(\left[n_{k_{2}} \hat{\rho}(t)-\left(\mathrm{n}_{k_{2}}+1\right) \rho_{a a}(t)\right]|2\rangle\left\langle 1\left|-n_{k_{2}} \rho_{12}(t)\right| a\right\rangle\langle a|\right)+ \\
& p \sqrt{\gamma_{1} \gamma_{2}} e^{i\left(\omega_{2}-\omega_{1}\right) t}\left(\left[n_{k_{1}} \hat{\rho}(t)-\left(\mathrm{n}_{k_{1}}+1\right) \rho_{a a}(t)\right]|1\rangle\left\langle 2\left|-n_{k_{1}} \rho_{21}(t)\right| a\right\rangle\langle a|\right)+ \\
& \gamma_{2}\left(\left[\overline{\mathrm{n}}_{k_{2}} \hat{\rho}(\mathrm{t})-\left(\bar{n}_{k_{2}}+1\right) \rho_{a a}(t)\right]|2\rangle\langle 2|+| a\rangle\langle a|\left[\left(\overline{\mathrm{n}}_{k_{2}}+1\right) \hat{\rho}(\mathrm{t})-\overline{\mathrm{n}}_{k_{2}} \rho_{22}(\mathrm{t})\right]\right)+ \\
& \left.\gamma_{1}|1\rangle\left\langle 1\left|\left[\overline{\mathrm{n}}_{k_{1}} \hat{\rho}(\mathrm{t})-\left(\bar{n}_{k_{1}}+1\right) \rho_{a a}(t)\right]+\left[\left(\overline{\mathrm{n}}_{k_{1}}+1\right) \hat{\rho}(\mathrm{t})-\overline{\mathrm{n}}_{k_{1}} \rho_{11}(\mathrm{t})\right]\right| a\right\rangle\langle a|\right)+ \\
& p \sqrt{\gamma_{1} \gamma_{2}} e^{-i\left(\omega_{1}-\omega_{2}\right) t}\left(|1\rangle\left\langle 2\left|\left[n_{k_{2}} \hat{\rho}(t)-\left(\mathrm{n}_{k_{2}}+1\right) \rho_{a a}(t)\right]-n_{k_{2}} \rho_{21}(t)\right| a\right\rangle\langle a|\right)+ \\
& p \sqrt{\gamma_{1} \gamma_{2}} e^{-i\left(\omega_{2}-\omega_{1}\right) t}\left(|2\rangle\left\langle 1\left|\left[n_{k_{1}} \hat{\rho}(t)-\left(\mathrm{n}_{k_{1}}+1\right) \rho_{a a}(t)\right]-n_{k_{1}} \rho_{12}(t)\right| a\right\rangle\langle a|\right)+ \\
& \gamma_{2}\left(|2\rangle\left\langle 2\left|\left[\overline{\mathrm{n}}_{k_{2}} \hat{\rho}(\mathrm{t})-\left(\bar{n}_{k_{2}}+1\right) \rho_{a a}(t)\right]+\left[\left(\overline{\mathrm{n}}_{k_{2}}+1\right) \hat{\rho}(\mathrm{t})-\overline{\mathrm{n}}_{k_{2}} \rho_{22}(\mathrm{t})\right]\right| a\right\rangle\langle a|\right)
\end{aligned}
$$

Taking matrix elements from this operator equation and replacing $\rho_{12} \rightarrow \rho_{12} e^{i\left(\omega_{1}-\omega_{2}\right) t}$ we finally obtain:

$$
\begin{gathered}
\dot{\rho}_{11}=\gamma_{1}\left[\left(\bar{n}_{1}+1\right) \rho_{a a}-\bar{n}_{1} \rho_{11}\right]-\frac{p \sqrt{\gamma_{1} \gamma_{2}}}{2} \bar{n}_{2}\left[\rho_{21}+\rho_{12}\right] \\
\dot{\rho}_{22}=\gamma_{2}\left[\left(\bar{n}_{2}+1\right) \rho_{a a}-\bar{n}_{2} \rho_{22}\right]-\frac{p \sqrt{\gamma_{1} \gamma_{2}}}{2} \bar{n}_{1}\left[\rho_{12}+\rho_{21}\right] \\
\dot{\rho}_{12}=-\frac{1}{2}\left(\gamma_{1} \bar{n}_{1}+\gamma_{2} \bar{n}_{2}\right) \rho_{12}+i \Delta \rho_{12}+\frac{p \sqrt{\gamma_{1} \gamma_{2}}}{2}\left[\left(\bar{n}_{1}+\bar{n}_{2}+2\right) \rho_{a a}-\left(\bar{n}_{1} \rho_{11}+\bar{n}_{2} \rho_{22}\right)\right]
\end{gathered}
$$

Where $\bar{n}_{i}$ is the photon average occupation number $\overline{\mathrm{n}}_{i}=\frac{1}{\exp \left(\frac{E_{a i}}{k_{B} T_{S}}\right)-1}$ (for $\left.i=1,2\right), \Delta=\omega_{1}-\omega_{2}$ is the splitting of the levels $|1\rangle$ and $|2\rangle, p \sqrt{\gamma_{1} \gamma_{2}}$ is the cross-coupling that describe the effect of interference in decay 
paths from $|a\rangle$ to lower levels, and $p=\frac{\wp_{1 a} \cdot \wp_{2 a}}{\left|\wp_{1 a}\right|\left|\wp_{2 a}\right|}$ quantifies the angle between the $1 \rightarrow a$ and $2 \rightarrow a$ transition dipole moments.

\section{REFERENCES}

[1] M. A. Green, Y. Hishikawa, W. Warta, E. D. Dunlop, D. H. Levis, J. Hohl - Ebinger, and A. W. Y. Ho-Baillie, "Solar cell efficiency tables (version 50)," Prog Photovolt Res Appl, vol. 25, pp. 668-676, 2017.

[2] W. Shockley and H. J. Queisser, "Detailed balance limit of efficiency of p-n junction solar cells," J. Appl. Phys., vol. 32, pp. 510-519, 1961.

[3] J. F. Guillemoles, Future concepts for photovoltaic energy conversion, Fundamentals of Materials for Energy and Environmental Sustainability, Cambridge: Cambridge University Press, 2011, ch. 19, pp. 238-56.

[4] A. Luque and A. Mart' 1 , "Increasing the efficiency of ideal solar cells by photon induced transitions at intermediate levels," Phys. Rev. Lett., vol. 78, pp. 5014-5017, 1997.

[5] A. Mart'1 and A. Luque, "Fundamentals of Intermediate Band Solar Cells," in Next Generation of Photovoltaics: New Concepts, vol. 165, A.B. Cristobal, A. Mart'1, and A. Luque, Eds., Berlin, Germany: Springer, 2012, pp. 209-228.

[6] A. S. Brown and M. A. Green, "Impurity photovoltaic effect: Fundamental energy conversion efficiency limits," J. Appl. Phys., vol. 92, pp. 1329-36, 2002.

[7] A. Luque, P. G. Linares, E. Antolin, E. Canovas, C.D. Farmer, C.R. Stanley, and A. Marti, "Multiple levels in intermediate band solar cells," Appl. Phys. Lett., vol. 96, p. 013501, 2010.

[8] T. Nozawa and Y. Arakawa, "Detailed balance limit of the efficiency of multilevel intermediate band solar cells," Appl. Phys. Lett., vol. 98 , p.171108, 2011.

[9] I. Ramiro, A. Martí, E. Antolín, and A. Luque, "Review of experimental results related to the operation of intermediate band solar cells," IEEE Journal of Photovoltaics, vol. 4(2), pp. 736-748, 2014.

[10] Y. Okada, N. J. Ekins-Daukes, T. Kita, R. Tamaki, M. Yoshida, A. Pusch, O. Hess, C. C. Phillips, D. J. Farrell, K. Yoshida, N. Ahsan, Y. Shoji, T. Sogabe, and J. F. Guillemoles, "Intermediate-band solar cells: recent progress and future directions", Applied Physics Reviews, vol. 2(2), p. 021302, 2015.

[11] A. Luque, "Will we exceed 50\% efficiency in photovoltaics?," J. Appl. Phys., vol. 11, p. 031301, 2011.

[12] N. López, L.A. Reichertz, K.M. Yu, K. Campman, and W. Walukiewicz, "Engineering the electronic band structure for multiband solar cells," Phys. Rev. Lett., vol. 106(2), p. 028701, 2011.

[13] H. Castán, E. Pérez, H. García, S. Duenas, L. Bailón, J. Olea, D. Pastor, E. García- Hemme, M. Irigoyen, and G. González-Díaz, "Experimental verification of intermediate band formation on titanium-implanted silicon," J. Appl. Phys. vol. 113(2), p. 024104, 2013.

[14] A. Luque, A. Martí and C. Stanley, "Understanding intermediate-band solar cells," Nature Photonics, vol. 6, pp. 146-152, 2012.

[15] I. Ramiro, A. Martí," InTERmediate Band SOlar Cells: Present And future," Prog Photovolt Res Appl, pp. 1-9, 2020.

[16] N. E. GORJI, M. HOUSHMAND and S. S. DEHKORDI, "CONSTRUCTION COMPONENTS ENGINEERING IN INTERMEDIATE BAND SOLAR CELLS”, Modern Physics Letters B, Vol. 26, No. 14, 1250090, 2012.

[17] I. Ramiro, J. Villa, J. Hwang, A.J. Martin, J. Millunchick, J. Phillips, A. Martí, "Demonstration of a GaSb/GaAs Quantum Dot Intermediate Band Solar Cell Operating at Maximum Power Point". Phys. Rev. Lett. Vol. 125, 247703, 2020.

[18] S. Jenks and R. Gilmore, "Material selection for the quantum dot intermediate band solar cell," in Lecture Notes in Nanoscale Science and Technology, pp. 135-166, Springer, New York, NY, USA, 2013.

[19] T. Kada et al., "Two-step photocarrier generation in InAs/GaAs quantum dot superlattice intermediate band solar cell," 2015 IEEE 42nd Photovoltaic Specialist Conference (PVSC), pp. 1-4, 2015.

[20] A. Mebadi, M. Houshmand, M.H. Zandi, N.E. Gorji, "Simulations of the intermediate bandwidth fluctuations in nanostructured" PV. Phys. E Low-Dimens. Syst. Nanostruct. Vol. 53, pp. 130-136, 2013.

[21] N. Cavassilas, D. Suchet, A. Delamarre, J.F. Guillemoles, M. Bescond, F. Michelini, M.Lannoo, "Physics of the inter-subband transition in quantum-dot intermediate-band solar cell," Proceedings Volume 11275, Physics, Simulation, and Photonic Engineering of Photovoltaic Devices IX; 112750Y,2020.

[22] S. E. Harris, "Lasers without Inversion Interference of Lifetime-Broadened Resonances," Phys. Rev. Lett., vol. 62, no. 9, pp. 10331036, 1989.

[23] S. E. Harris, "Electromagnetically Induced Transparency," Phys. Today, vol. 50, no. 7, pp. 36-42, 1997.

[24] L. V. Hau, S. E. Harris, Z. Dutton, and C. H. Behroozi, "Light speed reduction to 17 metres per second in an ultracold atomic gas," Nature, vol. 397, pp. 594-598, 1999.

[25] M. M. Kash,V. A. Sautenkov, A. S. Zibrov, L. Hollberg, G. R. Welch, M. D. Lukin, Y. Rostovtsev, E. S. Fry, and M. O. Scully, "Ultraslow Group Velocity and Enhanced Nonlinear Optical Effects in a Coherently Driven Hot Atomic Gas," Phys. Rev. Lett., vol. 82(26), p. 5229, 1999.

[26] W. W. Chow, H. C. Schneider, and M. C. Phillips, "Theory of quantum-coherence phenomena in semiconductor quantum dots," Phys. Rev. A, vol. 68(5), p. 053802, 2003.

[27] W. W. Chow, S. Michael, and H. C. Schneider, "Many-body theory of quantum coherence in semiconductor quantum dots," J. Mod. Opt., vol. 54(16-17), pp. 2413-2424, 2007.

[28] A. A. Belyanin, F. Capasso, V. V. Kocharovsky, Vl. V. Kocharovsky, and M. O. Scully, "Infrared generation in low-dimensional semiconductor heterostructures via quantum coherence," Phys. Rev. A, vol. 63(5), p.053803, 2001.

[29] M. O. Scully, "Quantum Photocell: Using Quantum Coherence to Reduce Radiative Recombination and Increase Efficiency," Phys. Rev. Lett., vol. 104(20), p. 207701, 2010.

[30] M.O. Scully, K.R. Chapin, K.E. Dorfman, M.B. Kim, and A. Svidzinsky, "Quantum heat engine power can be increased by noiseinduced coherence," PNAS. USA, vol. 108(37), pp. 15097-15100, 2011.

[31] A. Svidzinsky, K. Dorfman, and M. O. Scully, "Enhancing photovoltaic power by Fano-induced coherence," Phys. Rev. A, vol. 84(5), p. 053818, 2011. 
[32] K.E. Dorfman, D.V. Voronine, S. Mukamel, and M.O. Scully, "Photosynthetic reaction center as a quantum heat engine," PNAS. USA, vol. 110 (8), pp. 2746-2751, 2013.

[33] M. Fleischhauer, C. H. Keitel, M. O. Scully, and C. Su, "Lasing without inversion and enhancement of the index of refraction via interference of incoherent pump processes," Opt. Commun., vol. 87(3), pp. 109-114, 1992.

[34] G. S. Agarwal, "Quantum Statistical Theories of Spontaneous Emissions and Their Relation to Other Approaches," Springer Tracts in Modern Physics, vol. 70, Berlin, Springer, 1974.

[35] V. V. Kozlov, Y. Rostovtsev, and M. O. Scully, "Inducing quantum coherence via decays and incoherent pumping with application to population trapping, lasing without inversion, and quenching of spontaneous emission," Phys. Rev. A, vol. 74(6), p. 063829, 2006.

[36] C. Creatore, M. A. Parker, S. Emmott, and A. W. Chin, "Efficient Biologically Inspired Photocell Enhanced by Delocalized Quantum States," Phys. Rev. Lett., vol. 111(25), p. 253601, 2013.

[37] U. Fano, "Effects of Configuration Interaction on Intensities and Phase Shifts," Phys. Rev., vol. 124, no. 6, pp. 1866-1878, 1961.

[38] K.E. Dorfman, P.K. Jha, and S. Das, "Quantum-interference-controlled resonance profiles from lasing without inversion to photodetection," Phys. Rev. A, vol. 84(5), p. 053803, 2011.

[39] K. E. Dorfman, M. B. Kim, and A. A. Svidzinsky, "Increasing photocell power by quantum coherence induced by external source," Phys. Rev. A, vol. 84(5), p. 053829, 2011.

[40] A. A. Svidzinsky, K. E. Dorfman, and M. O. Scully, "Enhancing photocell power by noise-induced coherence," Coherent Optical Phenomena 1, pp. 7-24, 2012.

[41] K. E. Dorfman, A. A. Svidzinsky, and M.O. Scully, "Increasing Photovoltaic Power by Noise Induced Coherence Between Intermediate Band States," Coherent Optical Phenomena 1, pp. 42-49, 2013.

[42] M. Daryani, A. Rostami, G. Darvish, and M. K. Morravej-Farshi, "High efficiency solar cells using quantum interferences," Opt. Quant. Electron, vol. 49, p.255, 2017.

[43] B.-Q. Ou, L.-M. Liang, and C.-Z. Li, "Coherence-induced by incoherent pumping field and decay process in three-level $\Lambda$ type atomic system," Opt. Commun., vol. 281(19), pp. 4940-4945, 2008.

[44] M.O. Scully and M.S. Zubairy, Quantum Optics, Cambridge, UK: Cambridge University Press, 1997.

[45] K. Hoki and P. Brumer, "Excitation of Biomolecules by Coherent vs. Incoherent Light: Model Rhodopsin Photoisomerization," Procedia Chem., vol. 3(1), pp. 122-131, 2011.

[46] Z. S. Sadeq and P. Brumer, "Transient quantum coherent response to a partially coherent radiation field," J. Chem. Phys., vol. 140, p. 074104, 2014.

[47] T. Uzer (with an appendix by W. H. Miller), "Theories of intramolecular vibrational energy transfer," Phys. Rep., vol. 199(2), pp. 73-146, 1991. 